\title{
Production of neutron-rich nuclides in the vicinity of $N=126$ shell closure in multinucleon transfer reactions
}

\author{
Alexander Karpov ${ }^{1,2, \star}$ and Vyacheslav Saiko ${ }^{1,2, \star \star}$ \\ ${ }^{1}$ Flerov Laboratory of Nuclear Reactions, JINR, 141980 Dubna, Russia \\ ${ }^{2}$ Dubna State University, 141980 Dubna, Russia
}

\begin{abstract}
Multinucleon transfer in low-energy nucleus-nucleus collisions is widely discussed as a method of production of yet-unknown neutron-rich nuclei hardly accessible (or inaccessible) by other methods. Modeling of complicated dynamics of nuclear reactions induced by heavy ions is done within a multidimensional dynamical model of nucleus-nucleus collisions based on the Langevin equations. The model gives a continuous description of the system evolution starting from the well-separated target and projectile in the entrance channel of the reaction up to the formation of final reaction products. In this paper, rather recent sets of experimental data for the ${ }^{136} \mathrm{Xe}+{ }^{198} \mathrm{Pt},{ }^{208} \mathrm{~Pb}$ reactions are analyzed together with the production cross sections for neutron-rich nuclei in the vicinity of the $N=126$ magic shell.
\end{abstract}

\section{Motivation}

Nowadays, production and study of neutron-rich nuclei is one of the main trends in nuclear physics. A number of facilities over the world are already working on this subject. These studies, in part, are motivated by a quantitative understanding of the astrophysical $r$-process, which goes through neutron-rich nuclei far away from the betastability line. Such nuclides located in the vicinity of the neutron closed shells form the so-called $r$-process waiting points. Knowing the properties of these nuclides plays a key role in the modeling of the $r$-process.

One of the least explored neutron-rich areas of the nuclear chart is that close to the $N=126$ shell closure. That is caused by the low values of the fragmentation cross sections - the only method of production of neutron-rich nuclei in this area used so far. During the last decade, 34 new nuclides have been added to each of the isotonic chains in the vicinity of $N=126$ by ${ }^{238} \mathrm{U}$ and ${ }^{208} \mathrm{~Pb}$ fragmentation. Thus, the most neutron-enriched nucleus with $N=126$ known at present, ${ }^{202} \mathrm{Os}$, was produced $[1,2]$ with the cross section $4.4 \pm 2.0 \mathrm{pb}$ [2]. Moreover, only the lower limit of the ${ }^{202}$ Os half-life was identified. Since the fragmentation cross sections will continue their rapid decrease with each step in the direction of neutron excess, alternative efficient methods of production of these nuclei are still of great value.

Multinucleon transfer in the processes of deep inelastic (DI) collisions of heavy ions at near-barrier energies has been considered to be such an appropriate method since the discovery of this type of nuclear reactions in 1966 by V.V. Volkov with collaborators [3]. During several years

\footnotetext{
$\star^{\star}$ e-mail: karpov@jinr.ru

$\star \star$ e-mail: saiko@jinr.ru
}

after the discovery, a number of light neutron-rich nuclei were produced for the first time in DI collisions of light ions with heavy targets (see [4] and references therein).

A recently renewed interest in the DI processes is caused, in part, by a widely discussed possibility of synthesizing the unknown neutron-rich isotopes of mediummass, heavy, and superheavy nuclei [5-7, 13, 14]. A number of experimental studies have already been performed [8-12], aiming at the validation of this idea and studying the peculiarities of the multinucleon transfer reactions in this mass region.

Semiclassical approaches based on the Langevin equations are proved to be powerful tools for analysis of low-energy nucleus-nucleus collisions, allowing one to model low-energy collisions of heavy ions in their complexity of intersecting and competing reaction channels. In this work we analyze new experimental data on the ${ }^{136} \mathrm{Xe}+{ }^{198} \mathrm{Pt},{ }^{208} \mathrm{Pt}[8-10]$ reactions within a recently developed multidimensional dynamical model [15] which is based on the same principles as the one in Ref. [13]. The model is briefly described in the next section. Finally, the optimal conditions for production of neutron-rich nuclei with $N=126$ in multinucleon transfer reactions are discussed.

\section{Model}

A near-barrier collision of two nuclei may be described as consisting of three main stages: approaching stage, mononucleus formation, and separation into final fragments. It is necessary to realistically describe the system evolution at all these stages. The first step on this way is the choice of the most important degrees of freedom of the interacting system. Their number should be large enough to repro- 


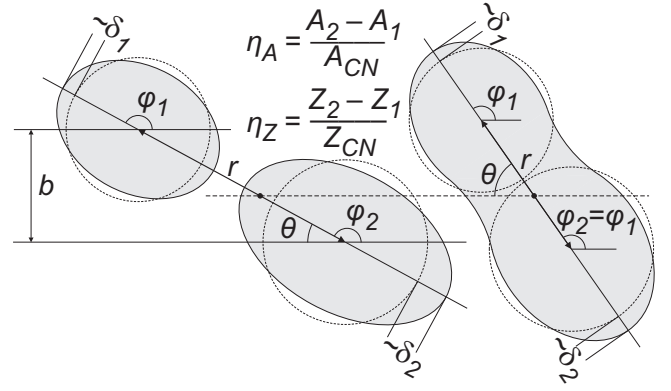

Figure 1. Schematic view of the nuclear degrees of freedom.

duce the main features of the process. On the other hand, we are limited by the present computational possibilities. The developed model has eight degrees of freedom: distance $r$ between the centers of two nuclei, two ellipsoidal surface deformations $\delta_{i}(i=1,2)$, mass and charge asymmetries $\eta_{A}, \eta_{Z}$, two angles $\varphi_{i}$ of rotation of the nuclei, and angle $\theta$ between the symmetry axis and beam direction. They are schematically shown in Fig. 1.

Potential energy, inertia and friction tensors are the main quantities regulating the nuclear dynamics. We calculate them on a grid, before starting the dynamical calculations. The potential energy takes into account the transition from the diabatic regime to adiabatic regime of nuclear interaction as a relaxation process with the characteristic time $\tau_{D A}[16]$ :

$$
\begin{array}{r}
V\left(r, \delta_{1}, \delta_{2}, \eta_{A}, \eta_{Z} ; t\right)=V_{\text {diab }} \exp \left(-\frac{t}{\tau_{D A}}\right)+ \\
+V_{\text {adiab }}\left[1-\exp \left(-\frac{t}{\tau_{D A}}\right)\right] .
\end{array}
$$

The diabatic potential is calculated using the doublefolding method with the Migdal nucleon-nucleon forces. The adiabatic potential energy is calculated within the macro-microscopic approach

$$
\begin{aligned}
V_{\mathrm{adiab}}\left(r, \delta_{1}, \delta_{2}, \eta_{A}, \eta_{Z}\right) & =V_{\mathrm{mac}}\left(r, \delta_{1}, \delta_{2}, \eta_{Z}, \eta_{A}\right)+ \\
& +\delta E\left(r, \delta_{1}, \delta_{2}, \eta_{Z}, \eta_{A}\right),
\end{aligned}
$$

where $V_{\mathrm{mac}}$ is the macroscopic part of the potential calculated within the finite-range liquid-drop model [17], $\delta E$ is the shell correction calculated of the basis of the twocenter shell model $[16,18]$. The details on the potential energy calculations may be found in Refs. [16, 19].

A rather traditional approach based on the Langevin equations was applied for the modeling of the time evolution of the nuclear system. The corresponding system of equations reads:

$$
\left\{\begin{array}{l}
\dot{q}_{i}=\mu_{i j} p_{j}, \\
\dot{p}_{i}=F_{i}^{\text {driving }}+F_{i}^{\text {friction }}+F_{i}^{\text {random }}
\end{array}\right.
$$

where $q_{i}$ and $p_{i}$ are the collective degrees of freedom and their conjugate momenta, respectively, $m_{i j}$ is the mass tensor $\left\|\mu_{i j}\right\|=\left\|m_{i j}\right\|^{-1}$.f The driving, friction, and random forces are calculated by the following expressions:

$$
\begin{aligned}
F_{i}^{\text {driving }} & =T \frac{\partial S}{\partial q_{i}}, \quad S=2 \sqrt{a E^{*}}, \\
F_{i}^{\text {friction }} & =-\sum_{j, k} \gamma_{i j} \mu_{j k} p_{k}, \\
F_{i}^{\text {random }} & =\sum_{j} \theta_{i j} \xi_{j}(t) .
\end{aligned}
$$

Here $S$ is the entropy of the excited nuclear system, $a$ is the level density parameter, $E^{*}=E_{\text {tot }}-V-E_{\text {kin }}$ is the excitation energy, $E_{\text {tot }}$ is the total energy of the system, $E_{\text {kin }}$ is the kinetic energy stored in all collective degrees of freedom, $\xi_{i}(t)$ are the normalized random variables with Gaussian distribution $\left\langle\xi_{i}(t)\right\rangle=0,\left\langle\xi_{i}(t), \xi_{j}\left(t^{\prime}\right)\right\rangle=2 \delta_{i j} \delta\left(t-t^{\prime}\right)$, and $\theta_{i j}$ are the amplitudes of the random force determined from the Einstein equation $\theta_{i k} \theta_{k j}=\gamma_{i j} T, T=\sqrt{E^{*} / a}$ is the nuclear temperature, and $\gamma_{i j}$ is the friction tensor.

Since the deformations, charge and mass asymmetries are finite collective modes, one should use an effective temperature $T_{i}^{\text {eff }}$, which takes into account quantum fluctuations, instead of the heat-bath temperature $T$ for amplitudes of the random force $\theta_{\delta_{i} \delta_{i}}, \theta_{\eta_{Z} \eta_{Z}}$, and $\theta_{\eta_{A} \eta_{A}}$ [20]

$$
T_{i}^{\mathrm{eff}}=\frac{\hbar \omega_{i}}{2} \operatorname{coth}\left(\frac{\hbar \omega_{i}}{2 T}\right),
$$

where $\omega_{i}$ is the frequency with respect to the mode $i=$ $\left\{\delta_{1}, \delta_{2}, \eta_{Z}, \eta_{A}\right\}$. Asymptotically, $T_{i}^{\text {eff }} \simeq T$ when $T \gg \hbar \omega_{i}$ and $T_{i}^{\text {eff }}=\hbar \omega_{i} / 2$ when $T=0$. It is clear that the use of the effective temperature instead of the thermodynamic one is extremely important at the first, approaching, stage of nucleus-nucleus collisions, when the temperature $T$ is close to zero. This, in particular, simulates the zerovibrations of the target and projectile, as well as mass and charge transfer between the not yet excited reaction partners. The difference in the use of $T_{i}^{\text {eff }}$ and $T$ gradually disappears, when the energy dissipates and the system gets excited. For the charge and mass asymmetry degrees of freedon, the use of the effective temperature is important even for well-excited systems due to large values of $\hbar \omega_{\eta_{i}}$ (up to several $\mathrm{MeV}$ ).

The inertia tensor components are calculated within the Werner-Wheeler approach for incompressible irrotational flow [21] as functions of the collective degrees of freedom: $m_{i j}$ where $\{i, j\}=\left\{r, \delta_{1}, \delta_{2}, \eta_{A}, \eta_{Z}\right\}$. The inertia coefficients for the angular degrees of freedom are the corresponding inertia moments. The one-body dissipation mechanism [22, 23] of nuclear viscosity is used for the friction tensor $\gamma_{i j}$ calculation. The friction coefficient $\gamma_{r r}$ calculated within the one-body friction model goes to zero for separated nuclei. However, the energy dissipation may start even before the contact. In order to account for this effect, we introduce a phenomenological nuclear friction force with a Woods-Saxon-type form factor (the friction strength depends on the amplitude $\gamma_{r}^{0}$ ). Thus, the friction force in the $r$ direction is the weighted sum of these two friction forces (see, e.g., Ref. [13]). Similarly, we introduce the dissipation and inertia coefficients for the mass and charge asymmetries, which are necessary for modeling nucleon transfer between the colliding (not yet touching) nuclei. 
Numerical solution of the Langevin equations (3) starts from the approaching stage of collision, when the target and projectile are separated by $50 \mathrm{fm}$, and finishes, when two reaction products are formed and separated again by an approximately $50 \mathrm{fm}$ distance. Each solution of these equations is a trajectory in the space of collective coordinates. It provides full information about a single collision, such as: charges and masses of primary (excited) reaction products, their kinetic energies, scattering angles, reaction time, etc. In order to study the characteristics of final fragments we use the statistical model of de-excitation of an excited rotating nucleus [19].

In this approach, the differential cross sections are calculated as follows: (i) a large number of trajectories at different impact parameters $0<b<b_{\max }$ are simulated; (ii) additional limitations on energies, angles, etc. are imposed according to the experimental measurement conditions; (iii) the differential cross sections are then calculated in a standard way, for example:

$$
\frac{d^{2} \sigma}{d E d \Omega}(E, \theta)=\int_{0}^{b_{\max }} \frac{\Delta N(b, E, \theta)}{N_{\mathrm{tot}}(b)} \frac{b d b}{\sin \theta \Delta \theta \Delta E},
$$

where $\Delta N$ is a number of trajectories in specific energy and angle bins and $N_{\text {tot }}$ is the total number of simulated trajectories for each impact parameter.

\section{Results and discussions}

The ${ }^{136} \mathrm{Xe}+{ }^{208} \mathrm{~Pb}$ reaction has been studied recently in Ref. [8] at several energies. The covered angular range was $25^{\circ} \leq \theta_{\mathrm{lab}} \leq 70^{\circ}$. Both reaction products were detected in coincidence using the time-of-flight method. This allows one to determine the characteristics of the primary (excited) reaction fragments that have survived against sequential fission. The experimental resolution (FWHM) is 7 units for the fragment mass and 25 units for each of the fragment energies. These experimental conditions and uncertainties are taken into account in the calculations.

Figure 2 shows the comparison of the experimental [8] and calculated energy, mass, and angular distributions for the primary fragments of the ${ }^{136} \mathrm{Xe}+{ }^{208} \mathrm{~Pb}$ reaction at two incident energies $E_{\mathrm{c} . \mathrm{m} .}=526$ and $617 \mathrm{MeV}$. In addition, the total mass yields of final fragments (including the events of sequential fission) are calculated without any restrictions and assuming ideal mass resolution (thin histograms). A good overall agreement with the data should be mentioned. An evident but not strong underestimation of the maximum value of the TKEL for higher collision energy should be the subject for further model tune.

It is also interesting to compare the calculations with the data not only for the integral mass yields but also for the independent isotopic yields. Such a comparison is shown in Fig. 3 for the isotopes of Po, Rn, and Ra determined in two independent experiments $[8,11]$ at two rather close collision energies. The data in Ref. [8] were accumulated in the $45^{\circ} \leq \theta_{\text {lab }} \leq 55^{\circ}$ angular range, while the entire solid angle was covered in Ref. [11]. The dashed histograms show the primary fragments yields. Since the
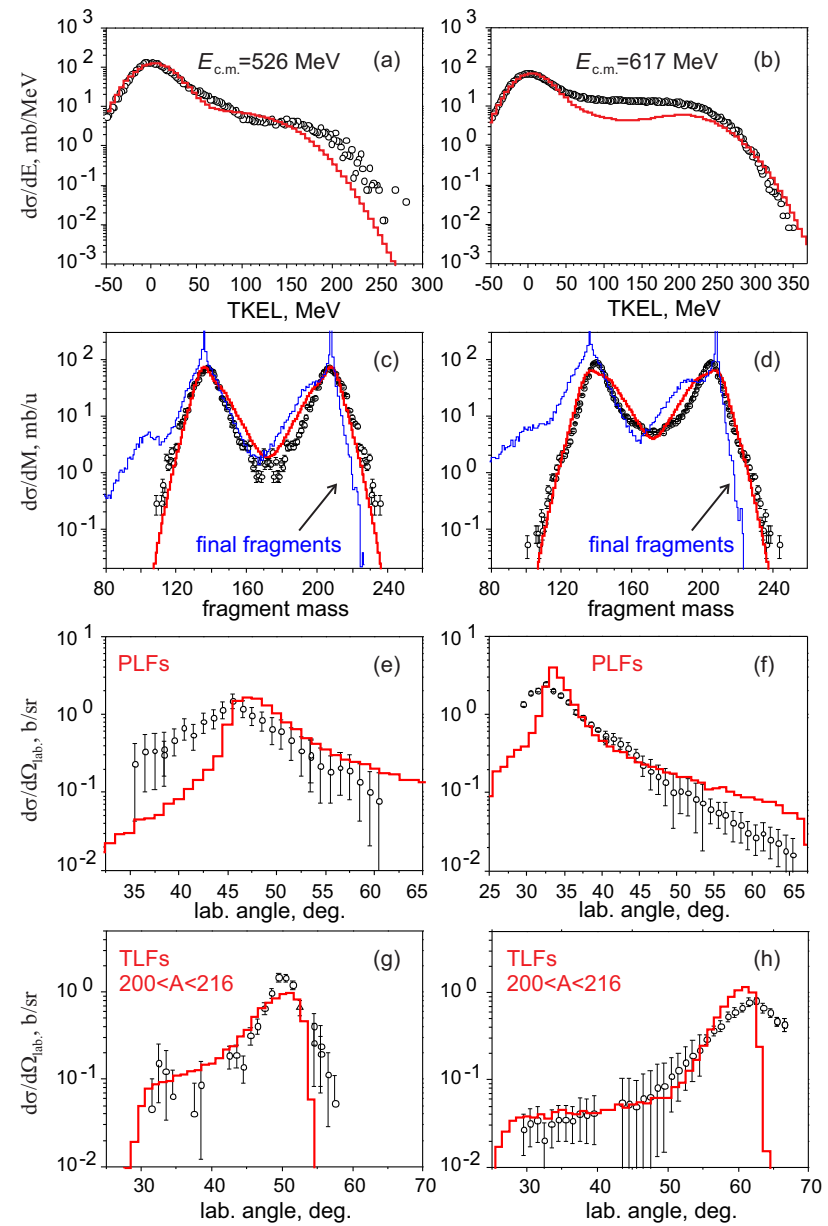

Figure 2. Energy, mass, and angular distributions of primary fragments for the ${ }^{136} \mathrm{Xe}+{ }^{208} \mathrm{~Pb}$ reaction for two collision energies $E_{\text {c.m. }}=526$ and $617 \mathrm{MeV}$. Experimental data (symbols) are from Ref. [8]. The angular distributions are shown for light reaction fragments $(A \leq 172)$ and heavy fragments with masses $200<A<216$. The thin histograms are the calculated mass distributions of final fragments including the sequential fission events.

de-excitation process for $E_{\mathrm{c} . \mathrm{m} .}=450 \mathrm{MeV}$ goes predominantly by neutron evaporation, the final fragment yields (solid histograms) represent the nearly-parallel-shifted primary ones. For higher collision energy, the excitation is large enough to have a substantial probability of sequential fission for isotopes of radon and radium. The calculations are in a good agreement with the data for proton-enriched nuclei, while they distinctly deviate from the data on the neutron-rich side for $E_{\mathrm{c} . \mathrm{m} .}=450 \mathrm{MeV}$ and reproduce the data for $E_{\text {c.m. }}=514 \mathrm{MeV}$.

An even more interesting for this particular paper comparison can be made for the production of osmium isotopes in the same projectile-target combination. This domain of nuclides is located in the vicinity of $N=126$. It is this domain that we intend to study. The corresponding experiments were performed at $E_{\text {c.m. }}=450 \mathrm{MeV}$ in Ref. [11] and at $E_{\text {c.m. }}=496 \mathrm{MeV}$ in Ref. [9]. In the later case, osmium isotopes having kinetic energies in the $400<E(\mathrm{Os})<600 \mathrm{MeV}$ range were registered for 

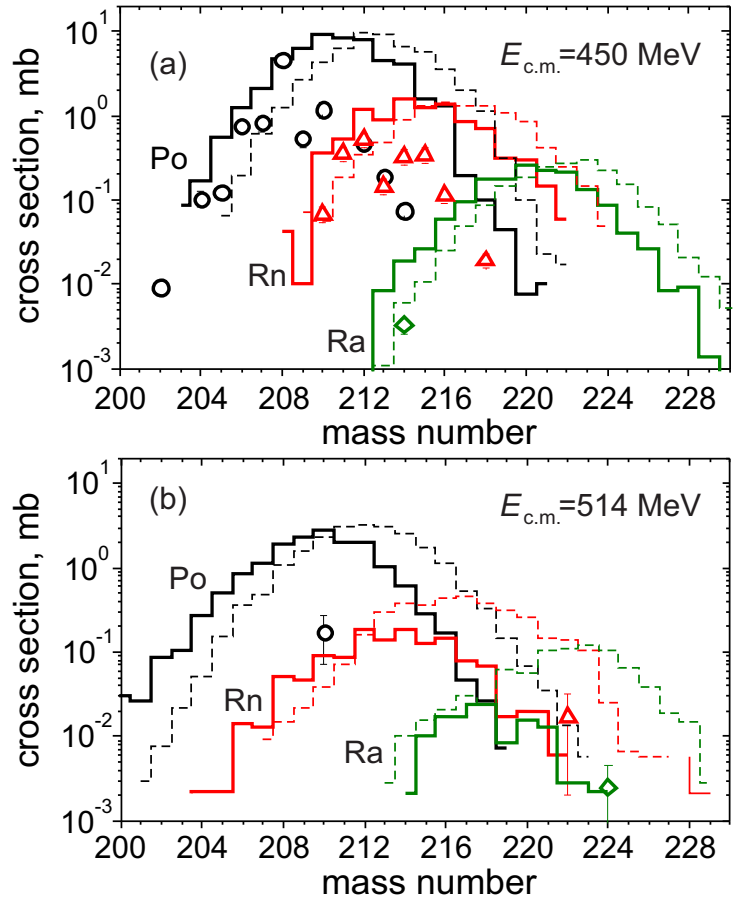

Figure 3. Calculated primary (dashed histograms) and final (solid histograms) yields of Po, Rn, and Ra isotopes for the ${ }^{136} \mathrm{Xe}+{ }^{208} \mathrm{~Pb}$ reaction in comparison with experimental data (circles, triangles, and diamonds for Po, Rn, and $\mathrm{Ra}$, respectively) taken from Ref. [11] for $E_{\text {c.m. }}=450 \mathrm{MeV}$ and Ref. [8] for $E_{\mathrm{c} . \mathrm{m} .}=514 \mathrm{MeV}$.

$31^{\circ} \leq \theta_{\mathrm{lab}} \leq 54^{\circ}$. The calculated cross sections are compared with the data in Fig. 4. The data shown in Fig. 9 of Ref. [9] are not absolute cross sections but relative yields, which have been normalized to the theoretical predictions of Ref. [24]. We have normalized these data to the present calculations (the data of Ref. [9] have been multiplied by ten). Thus, one may compare only the slopes of the calculated and measured cross sections. A good agreement of the calculations with the data for $E_{\mathrm{c} . \mathrm{m} .}=496 \mathrm{MeV}$ should be noticed. As in the case of Po, Rn, and Ra isotopes, overestimation of the cross sections for production of neutron-rich nuclides is seen for the data of Ref. [11]. This suggests that additional studies (both theoretical and experimental) are required.

In order to further verify the model and following the suggestion of L. Corradi made during the FUSION17 conference, the experimental data for the ${ }^{136} \mathrm{Xe}+{ }^{198} \mathrm{Pt}$ system at $E_{\text {c.m. }}=643 \mathrm{MeV}$ [10] have been analyzed. The results are summarized in Fig. 5 for the projectile-like fragments (PLF) and in Fig. 6 for the osmium and mercury isotopes. In the later case, the data accumulated in different TKEL gates are shown as well. The cross sections are integrated over the experimentally covered angles from $24^{\circ}$ to $34^{\circ}$. The calculations reproduce all the data for this system quite well. A shift (maximum of three mass units) of the calculations with respect to the data for the PLF towards neutron-richness is however seen for larger proton transfers.
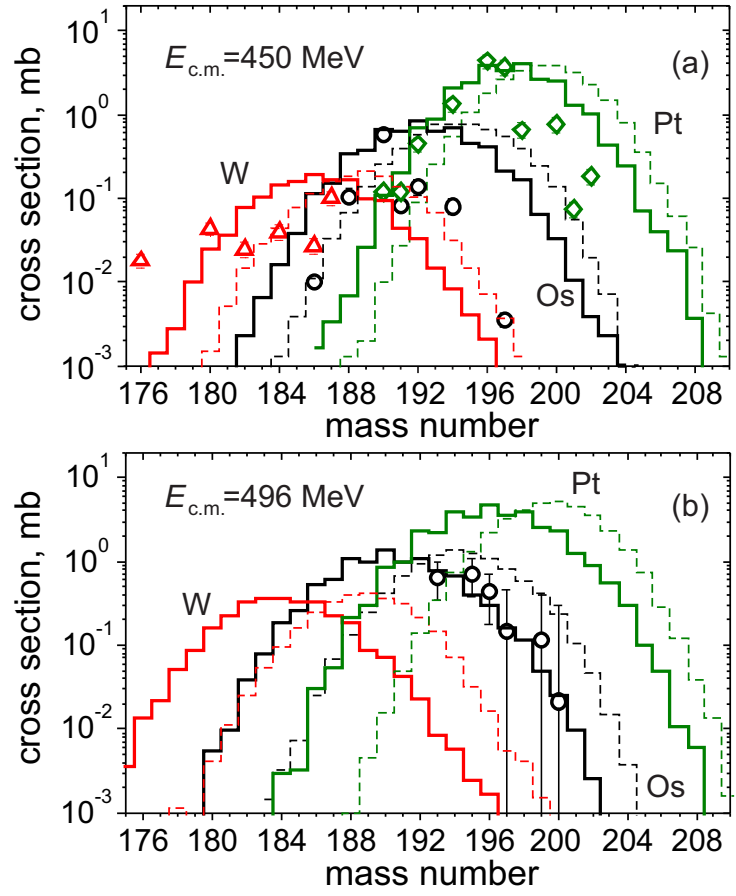

Figure 4. Same as in Fig. 3 but for W, Os, and Pt isotopes. The data (triangles, circles, and diamonds are for $\mathrm{W}$, Os, and Pt, respectively) are taken from Ref. [11] for $E_{\text {c.m. }}=450 \mathrm{MeV}$ and Ref. [9] for $E_{\text {c.m. }}=496 \mathrm{MeV}$. The data of Ref. [9] are multiplied by ten (see the text).

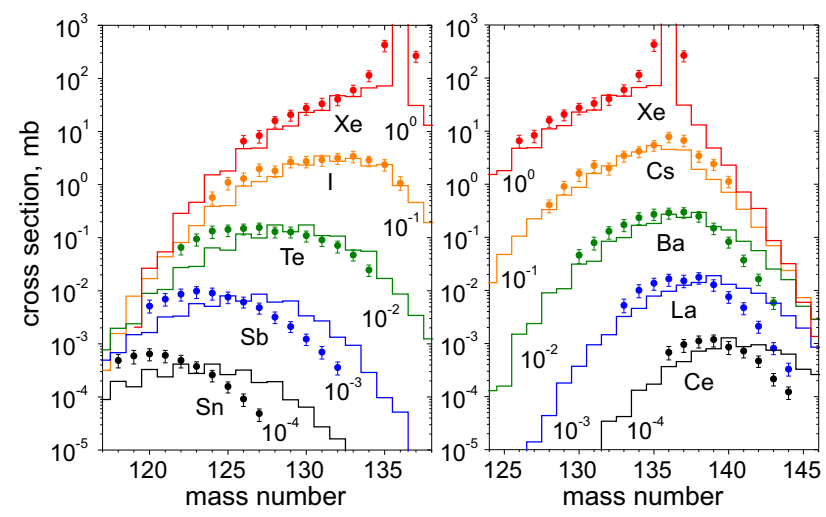

Figure 5. Calculated (histograms) and experimental [10] (symbols) cross sections of PLF for the ${ }^{136} \mathrm{Xe}+{ }^{198} \mathrm{Pt}$ reaction at $E_{\text {c.m. }}=643 \mathrm{MeV}$.

Finally, the data on the production cross sections of the $N=126$ nuclides in the ${ }^{136} \mathrm{Xe}+{ }^{198} \mathrm{Pt},{ }^{208} \mathrm{~Pb}$ reactions are compared with the calculations in Fig. 7 (a). Regarding the comparison with the experimental data, one may see a good agrement with the data of Ref. [10] and proton-rich nuclei from Ref. [11]. The calculated cross sections for production of neutron-rich nuclei in the ${ }^{136} \mathrm{Xe}+{ }^{208} \mathrm{~Pb}$ reaction strongly overestimate the data. According to the calculations, the cross sections are larger for the ${ }^{136} \mathrm{Xe}+{ }^{198} \mathrm{Pt}$ combination than for the ${ }^{136} \mathrm{Xe}+{ }^{208} \mathrm{~Pb}$ one. The difference increases with the increasing neutron-richness of a nuclide. The cross sections calculated at two different in- 

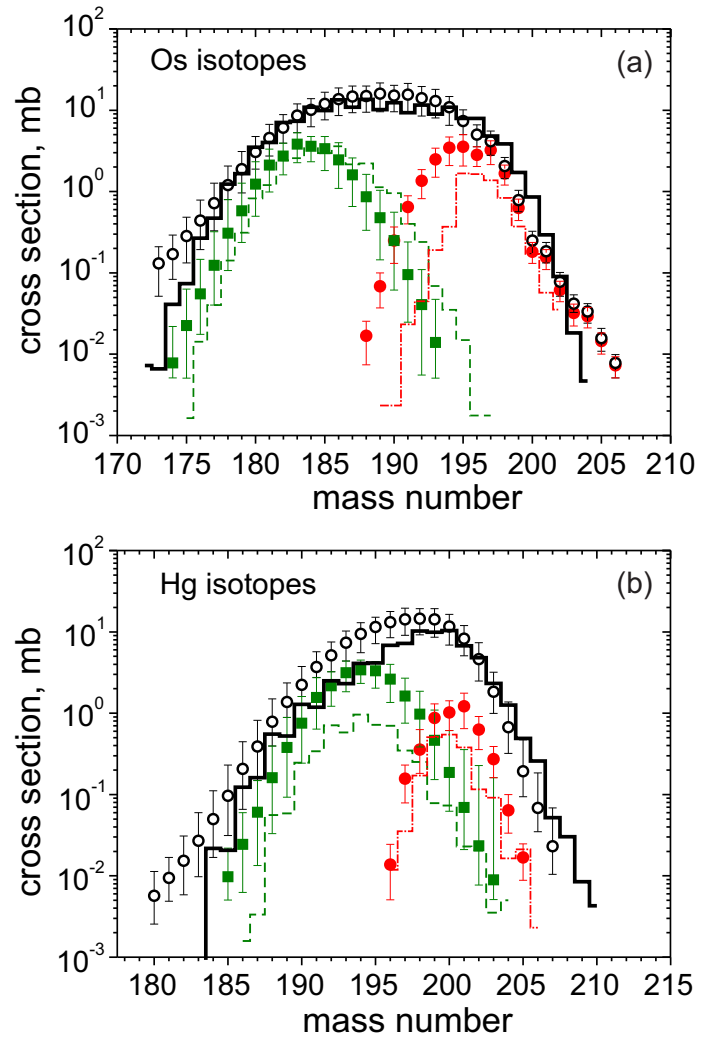

Figure 6. Calculated (solid histograms) and experimentally deduced in Ref. [10] (open circles) cross sections for $\mathrm{Hg}$ and Os isotopes integrated over the angular range from $24^{\circ}$ to $34^{\circ}$. Isotopic distributions for different ranges of TKEL from -25 to 25 $\mathrm{MeV}$ (dash-dotted histograms and filled circles) and from 175 to $225 \mathrm{MeV}$ (dashed histograms and filled squares) are shown.

cident energies for ${ }^{136} \mathrm{Xe}+{ }^{198} \mathrm{Pt}$ are quite close. Thus, an optimal energy should be searched for in future studies.

Rather important conclusions can be made regarding the optimal angular ranges for production of neutron-rich nuclei in multinucleon transfer reactions [see Fig. 7 (b)]. While the angular distributions are broad being integrated over an isotope mass (iridium in our case), they are much more localized for a specific neutron-rich isotope (the ${ }^{203} \operatorname{Ir}_{126}$ case is shown). The maximum for all the three studied reactions are close to the corresponding angles of grazing collisions. Obviously, the degree of localization depends on the system and incident energy. The sharpest angular distribution is predicted for the ${ }^{136} \mathrm{Xe}+{ }^{198} \mathrm{Pt}$ system at $E_{\text {c.m. }}=643 \mathrm{MeV}$. It is consistent with the calculations of cross sections [compare thick and thin histograms for this system in Fig. 7 (a)], as well as the data and calculations in Fig. 6. This figure shows that the cross sections for production of neutron-rich isotopes are almost exhausted by events having low TKEL that corresponds to peripheral collisions. Highly excited transfer products formed in central collisions do not survive. Low values of neutron binding energies amplify this effect. At lower near-barrier energies, even central collisions lead to low excitation energies of primary products (see difference of the primary and final cross sections shown in Fig. 4 for two collision energies). Moreover, the interac-
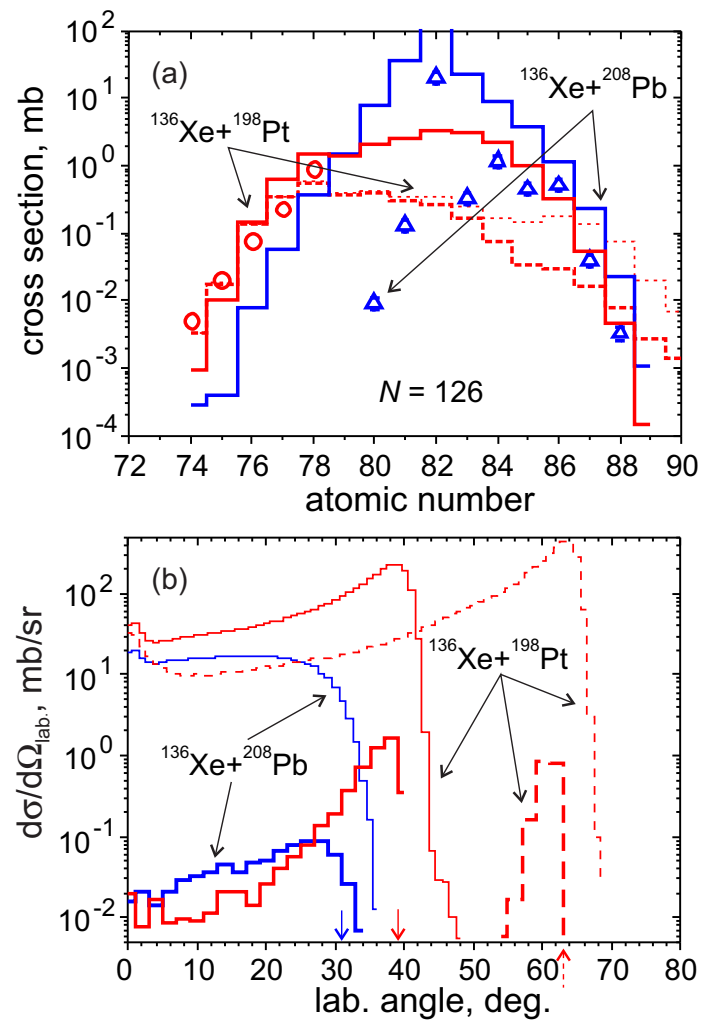

Figure 7. (a): Calculated (histograms) and experimental (symbols) cross sections for production of isotopes with $N=126$ in the ${ }^{136} \mathrm{Xe}+{ }^{198} \mathrm{Pt},{ }^{208} \mathrm{~Pb}$ reactions. The solid and dashed histograms are for $E_{\text {c.m. }}=450$ and $643 \mathrm{MeV}$, respectively. The thin dashed curve is integrated over all angles and the thick one - over the experimentally covered angles from $24^{\circ}$ to $34^{\circ}$. The experimentally deduced cross sections for the ${ }^{136} \mathrm{Xe}+{ }^{198} \mathrm{Pt}$ system is from Ref. [10] and for ${ }^{136} \mathrm{Xe}+{ }^{208} \mathrm{~Pb}$ is from Ref. [11]. (b): Calculated angular distributions in the laboratory frame for the Ir isotopes for the ${ }^{136} \mathrm{Xe}+{ }^{198} \mathrm{Pt},{ }^{208} \mathrm{~Pb}$ reactions. The thick histograms are for the ${ }^{203} \operatorname{Ir}_{126}$ nucleus and the thin histograms are integrated over all iridium isotopes. The solid and dashed histograms are for $E_{\text {c.m. }}=450$ and $643 \mathrm{MeV}$, respectively. The vertical arrows indicate the corresponding grazing angles.

tion time increases with the decreasing impact parameter that stimulates nucleon transfer. Thus, the angular distributions for neutron-rich nuclides become broader extending towards the forward angles. The most uniform distribution is obtained for the ${ }^{136} \mathrm{Xe}+{ }^{208} \mathrm{~Pb}$ combination at $E_{\text {c.m. }}=450 \mathrm{MeV}$ that can be explained by the lowest energy above the barrier (contact point potential). This peculiarities should be taken into account, while constructing experimental setups for studying multinucleon transfer reactions and planning the corresponding experiments.

Figure 8 shows the contour map of the production cross sections for ${ }^{136} \mathrm{Xe}+{ }^{208} \mathrm{~Pb}\left(E_{\text {c.m. }}=450 \mathrm{MeV}\right)$. It is seen that the last shown line corresponding to $100 \mathrm{nb}$ follows the border of the known neutron-rich nuclei in a wide range of atomic numbers. The most neutron-rich nuclides in the $N=126$ area can be synthesized in this reaction with the cross sections exceeding $100 \mathrm{nb}$, that is more than five orders of magnitude higher than one may expect for the fragmentation reactions used so far. 


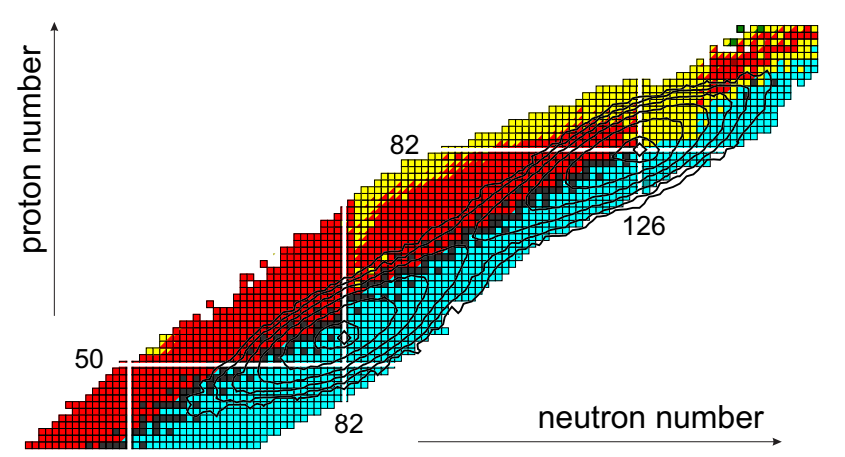

Figure 8. Upper part of the chart of nuclides. The contour lines are the multinucleon transfer cross sections for the ${ }^{136} \mathrm{Xe}+{ }^{208} \mathrm{~Pb}$ system at $E_{\text {c.m. }}=450 \mathrm{MeV}$. The contour lines are drawn over each order of the cross section down to $100 \mathrm{nb}$.

To conclude, the developed dynamical model of nucleus-nucleus collisions has shown its applicability for deep inelastic reactions. The performed calculations allows us to make rather optimistic conclusions about the perspectives of using the multinucleon transfer reactions for production of neutron-rich nuclei in the vicinity of the $N=126$ shell closure.

\section{References}

[1] S.J. Steer, Zs. Podolyák, S. Pietri, M. Górska, G.F. Farrelly, P.H. Regan, D. Rudolph, A.B. Garnsworthy, R. Hoischen, J. Gerl, H.J. Wollersheim, H. Grawe, K.H. Maier, F. Becker, P. Bednarczyk, L. Cáceres, P. Doornenbal, H. Geissel, J. Grebosz, A. Kelic, I. Kojouharov, N. Kurz, F. Montes, W. Prokopowicz, T. Saito, H. Schaffner, S. Tashenov, A. Heinz, T. Kurtukian-Nieto, G. Benzoni, M. Pfützner, A. Jungclaus, D.L. Balabanski, C. Brandau, A. Brown, A.M. Bruce, W.N. Catford, I.J. Cullen, Zs. Dombrádi, M.E. Estevez, W. Gelletly, G. Ilie, J. Jolie, G.A. Jones, M. Kmiecik, F.G. Kondev, R. Krucken, S. Lalkovski, Z. Liu, A. Maj, S. Myalski, S. Schwertel, T. Shizuma, P.M. Walker, E. Werner-Malento, and O. Wieland, Int. J. Mod. Phys. E 18, 1002 (2009).

[2] J. Kurcewicz, F. Farinon, H. Geissel, S. Pietri, C. Nociforo, A. Prochazka, H. Weick, J.S. Winfield, A. Estradé, P.R.P. Allegro, A. Bail, G. Bélier, J. Benlliure, G. Benzoni, M. Bunce, M. Bowry, R. CaballeroFolch, I. Dillmann, A. Evdokimov, J. Gerl, A. Gottardo, E. Gregor, R. Janik, A. Kelić-Heil, R. Knöbel, T. Kubo, Yu.A. Litvinov, E. Merchan, I. Mukha, F. Naqvi, M. Pfützner, M. Pomorski, Zs. Podolyák, P.H. Regan, B. Riese, M.V. Ricciardi, C. Scheidenberger, B. Sitar, P. Spiller, J. Stadlmann, P. Strmen, B. Sun, I. Szarka, J. Taïeb, S. Terashima, J.J. ValienteDobón, M. Winkler, and Ph. Woods, Phys. Lett. B 717, 371 (2012).

[3] A.G. Artukh, G.F. Gridnev, V.L. Mikheev, V.V. Volkov, and J. Wilczynski, Nucl. Phys. A 215, 91 (1973).
[4] V.V. Volkov, Phys. Rep. 44, 93 (1978); V.V. Volkov, Nuclear Reactions of High-Inelastic Transfers, Energoizdat, Moscow, 1982 (in Russian).

[5] C.H. Dasso, G. Pollarolo, and A. Winther, Phys. Rev. Lett. 73, 1907 (1994).

[6] R. Broda, J. Phys. G 32, R151 (2006).

[7] L. Corradi, G. Pollarolo, and S. Szilner, J. of Phys. G 36, 113101 (2009).

[8] E.M. Kozulin, E. Vardaci, G.N. Knyazheva, A.A. Bogachev, S.N. Dmitriev, I.M. Itkis, M.G. Itkis, A.G. Knyazev, T.A. Loktev, K.V. Novikov, E.A. Razinkov, O.V. Rudakov, S.V. Smirnov, W. Trzaska, and V.I. Zagrebaev, Phys. Rev. C 86, 044611 (2012).

[9] K. Novikov, E.M. Kozulin, I.M. Harca, S. Dmitriev, A. Flueras, P. Greenlees, F. Hanappe, S.V. Khlebnikov, T. Loktev, J. Maurer, A. Di Nitto, J. Pakarinen, P. Ruotsalainen, M. Sandzelius, J. Sorri, M. Sin, W.H. Trzaska, E. Vardaci, and V.I. Zagrebaev, J. Phys.: Conf. Ser. 515, 012016 (2014).

[10] Y.X. Watanabe, Y.H. Kim, S.C. Jeong, Y. Hirayama, N. Imai, H. Ishiyama, H.S. Jung, H. Miyatake, S. Choi, J.S. Song, E. Clement, G. de France, A. Navin, M. Rejmund, C. Schmitt, G. Pollarolo, L. Corradi, E. Fioretto, D. Montanari, M. Niikura, D. Suzuki, H. Nishibata, and J. Takatsu, Phys. Rev. Lett. 115, 172503 (2015).

[11] J.S. Barrett, W. Loveland, R. Yanez, S. Zhu, A.D. Ayangeakaa, M.P. Carpenter, J.P. Greene, R.V.F. Janssens, T. Lauritsen, E.A. McCutchan, A.A. Sonzogni, C.J. Chiara, J.L. Harker, and W.B. Walters, Phys. Rev. C 91, 064615 (2015).

[12] S. Heinz, O. Beliuskina, V. Comas, H.M. Devaraja, C. Heinz, S. Hofmann, E. Kozulin, F. Morherr, G. Münzenberg, D. Ackermann, F.P. Heßberger, B. Kindler, B. Lommel, R. Mann, and J. Maurer, Eur. Phys. J. A 51, 140 (2015).

[13] V.I. Zagrebaev and W. Greiner, J. Phys. G 34, 1 (2007).

[14] V.I. Zagrebaev and W. Greiner, J. Phys. G 35, 125103 (2007).

[15] A.V. Karpov and V.V. Saiko, Phys. Part. Nucl. Lett. (2017) in press.

[16] V. Zagrebaev, A. Karpov, Y. Aritomo, M. Naumenko, and W. Greiner, Phys. Part. Nucl. 38, 469 (2007).

[17] P. Möller, J.R. Nix, W.D. Myers, and W.J. Swiatecki, At. Data Nucl. Data Tables 59, 185 (1995).

[18] J. Maruhn and W. Greiner, Z. Phys. A 251, 431 (1972).

[19] Nuclear Reaction Video project, http://nrv.jinr.ru.

[20] L.D. Landau and E.M. Lifshitz, Statistical Physics. Part 1, Pergamon Press, Oxford, 1980.

[21] K.T.R. Davies, A.J. Sierk, and J.R. Nix, Phys. Rev. C 132385 (1976).

[22] A.J. Sierk and J.R. Nix, Phys. Rev. C 21, 982 (1980).

[23] A.V. Karpov and G.D. Adeev, Phys. At. Nucl. 65, 1596 (2002); Eur. Phys. J. A 14, 169 (2002).

[24] V.I. Zagrebaev and W. Greiner, Phys. Rev. Lett. 101, 122701 (2008). 